\title{
Modification Interior-Point Method for Solving Interval Linear Programming
}

\author{
Agustina Pradjaningsih", ${ }^{1, *}$, Fatmawati ${ }^{2}$, Herry Suprajitno $^{2}$ \\ ${ }^{1}$ Department of Mathematics, Faculty of Mathematics and Science, Jember University \\ ${ }^{2}$ Department of Mathematics, Faculty of Science and Technology, Universitas Airlangga \\ ${ }^{*}$ Corresponding author. Email: agustina.fmipa@unej.ac.id
}

\begin{abstract}
Linear programming is mathematical programming developed to deal with optimization problems involving linear equations in the objective and constraint functions. One of the basic assumptions in linear programming problems is the certainty assumption. Assumption of certainty shows that all coefficients variable or decision variables in the model are constants that are known with certainty. However, in real situations or problems, there may be uncertain coefficients or decision variables. Based on the concept and theory of interval analysis, this uncertainty problem is anticipated by making approximate values in intervals to develop linear interval programming. The development of interval linear programming starts from linear programming with interval-shaped coefficients, both in the coefficient of the objective function and the coefficient of the constraint function. It was subsequently developed into linear programming with coefficients and decision variables in intervals, commonly known as interval linear programming. Until now, the completion of interval linear programming is based on the calculation of the interval limit. The initial procedure for the solution is to change the linear programming model with interval variables into two classical linear programming models. Finally, the optimal solution in the form of intervals is obtained by constructing two models. This paper provides an alternative solution to directly solve the linear interval programming problem without building it into two models. The solution is done using the interval arithmetic approach, while the method used is the modified interior-point method.
\end{abstract}

Keywords: Interval Linear Programming, Interior Point Method, Interval Arithmetic.

\section{INTRODUCTION}

Linear programming is mathematical programming developed to handle optimization problems involving linear equations in the objective and constraint functions. Linear programming problems must satisfy the basic assumptions: proportionality, additive, divisible, and certainty. The certainty assumption shows that all the coefficients of the decision variables in the model are constants that are known with certainty. However, in real situations or problems, there may be uncertain coefficients or decision variables $[1,2]$.

One method for solving linear programming problems is using the interior-point method [3]. The first step to constructing an interior-point method is to transform the general form of classical linear programming represented in matrix form into a standard linear programming form [2]. The problems of linear and quadratic programming have used the interior-point method [4]. Therefore, as an alternative method of solving linear programming problems, the interior-point method needs a more comprehensive appreciation [5]. So far, the interior point method is used to solve classical linear programming problems that satisfy the assumption of certainty, with coefficients and variables in the form of constants [2]. Interval analysis can be used to anticipate this uncertainty [6]. Based on the concept and theory of interval analysis developed by [7], this uncertainty problem is anticipated by making approximate values in intervals to construct interval linear programming.

The development of interval linear programming starts from a linear program with coefficients in the form of intervals, both on the objective function's coefficient and the constraint function's coefficient. The optimum value of the objective function is obtained by combining the optimum value of the best optimum problem and the worst optimum problem so that it is in the form of an interval, while the optimum point is not in the form of an interval $[8,9,10,11]$. Furthermore, a linear program with coefficients in the form of intervals develops into a linear 
program with coefficients and decision variables in the form of intervals. In this development, the problem is solved by bringing into two classical linear programming models to get the optimum solution in intervals, both the optimum point and the optimum value $[12,13,14]$. According to $[13,15,16,17]$ used the simplex method to solve linear interval programming with an interval arithmetic approach. This interval arithmetic programming aims to get a solution for linear interval programming directly.

As a continuation of [14], this paper presents a solution for interval linear programming. The method used is an interior-point method that has been modified to solve linear interval programming directly through an arithmetic interval programming approach. Next, we will take examples from $[15,18,19,20]$ for the application of the modified solution.

\section{INTERVAL ARITHMETIC}

The basic concepts: definitions, properties of interval numbers, interval arithmetic, comparison of two intervals, and interval matrices can be found in $[7,9,15,21,22]$. Let $\mathrm{R}$ denote the set of all real numbers.

Definition 1. A closed real interval $\underline{x}=\left[x_{I}, x_{S}\right]$, is defined by

$\underline{x}=\left[x_{I}, x_{S}\right]=\left\{x_{I}, x_{S} \in \mathbb{R} \mid x_{I} \leq x_{S}\right\}$

where $x_{I}$ and $x_{S}$ are called infimum and supremum of $\underline{x}$, respectively.

Definition 2. A real interval $\underline{x}=\left[x_{I}, x_{S}\right]$, is called degenerate, if $x_{I}=x_{S}$.

Theorem 3. If $\left[x_{I}, x_{S}\right]=\left[y_{I}, y_{S}\right]$, then $x_{S} \geq y_{I}$ and $x_{I} \leq$ $y_{S}$

Definition 4. The width of an interval $\underline{x}$ is the real number $w(\underline{x})=\frac{1}{2}\left(x_{S}-x_{I}\right)$.

Definition 5. The midpoint of an interval $x$ is the real number $m(\underline{x})=\frac{1}{2}\left(x_{I}+x_{S}\right)$.

Definition 6. The absolute value of an interval $\underline{x}$ is a real numbe $|\underline{x}|=\operatorname{maks}\left\{\left|x_{I}\right|,\left|x_{S}\right|\right\}$.

Definition 7. Let $\underline{x}, \underline{y} \in I(\mathbb{R})$ where $\underline{x}=\left[x_{I}, x_{S}\right]$ and $\underline{y}=\left[y_{I}, y_{S}\right]$, then

1) addition :

$$
\underline{x}+\underline{y}=\left[x_{I}+y_{I}, x_{S}+y_{S}\right] \text {. }
$$

2) subtraction

$$
\begin{aligned}
& \underline{x}-\underline{y}=\left[x_{I}, x_{S}\right]-\left[y_{I}, y_{S}\right]=\left[x_{I}, x_{S}\right]+ \\
& {\left[-y_{S},-y_{I}\right]=\left[x_{I}-y_{S}, x_{S}-y_{I}\right] .}
\end{aligned}
$$

3) multiplication : $\underline{x y}$

$=\left[\min \left\{x_{I} y_{I}, x_{I} y_{S}, x_{S} y_{I}, x_{S} y_{S}\right\}, \operatorname{maks}\left\{x_{I} y_{I}, x_{I} y_{S}, x_{S} y_{I}, x_{S} y_{S}\right\}\right]$

4) division :

$$
\underline{\underline{x}}=\underline{x} \frac{1}{\underline{y}}=\left[x_{I}, x_{S}\right]\left[\frac{1}{y_{S}}, \frac{1}{y_{I}}\right], 0 \notin \underline{y}
$$

Definition 8. An interval vector $\underline{V} \in I\left(\mathbb{R}^{n}\right)$, is a set of the form $\underline{V}=\left(\underline{V_{i}}\right)_{n \times 1}$, where $\quad \underline{V_{i}}=\left[x_{i I}, x_{i S}\right] \in$ $I(\mathbb{R}), x_{i I}, x_{i S} \in \mathbb{R}$, and $i=1,2, \ldots, n$.

Definition 9. Let $\underline{x}, \underline{y} \in I(\mathbb{R})$ where $\underline{x}=\left[x_{I}, x_{S}\right]$ and $\underline{y}=\left[y_{I}, y_{S}\right]$, then

1) $\underline{x} \leq \underline{y}$ iff $x_{S} \leq y_{I}$.

2) $\underline{x} \leq \bar{y}$ iff $x_{I} \geq y_{I}$ and $x_{S} \leq y_{S}$.

3) $\underline{x} \leq \underline{y}$ iff $x_{I} \leq y_{I}$ and $x_{S} \leq y_{S}$.

4) a) $\underline{x} \leq \underline{y}$ iff $x_{I} \leq y_{I}$ and $m(x) \leq m(y)$.

b) $\underline{x} \leq \underline{y}$ iff $x_{S}>y_{I}$ and $m(x)<m(y)$.

c) $\underline{x} \leq \underline{y}$ iff $m(x) \leq m(y)$ and $w(x) \geq w(y)$.

5) a) $\underline{x} \leq y$ iff $x_{I}-\epsilon \leq y_{S}$ where $\epsilon$ real number.

b) $\underline{x} \leq \underline{y}$ iff $x_{S}-\epsilon \leq y_{I}$ where $\epsilon$ real number.

6) $\underline{x} \leq \underline{y}$ iff $x_{I}+x_{S} \leq y_{I}+y_{S}$.

7) $\underline{x} \leq \bar{y}$ iff $u x_{I}+v x_{S} \leq u y_{I}+v y_{S}$ where $u, v \in$ $(0,1]$ and $u \leq v$.

Definition 10. An interval matrix $\underline{\mathbf{A}} \in I\left(\mathbb{R}^{m \times n}\right)$, is a matrix $\underline{\mathbf{A}}=\left(a_{i j}\right)$ where $a_{i j}=\left[a_{i j l}, a_{i j S}\right] \in I(\mathbb{R})$, and $a_{i j I}$ is infimum $a_{i j}$ and $a_{i j S}$ is supremum $a_{i j}$, for every $i$ $=1,2, \ldots, m, j=1,2, \ldots, n$.

Definition 11. The midpoint of an interval matrix $\underline{\mathbf{A}}$ is the matrix $m \underline{\mathbf{A}})=\left(m\left(\underline{a_{i j}}\right)\right)$ where $m\left(\underline{a_{i j}}\right)=\frac{1}{2}\left(a_{i j I}+\right.$ $\left.a_{i j S}\right)$.

Definition 12. The width of an interval matrix $\underline{\mathbf{A}}$ is the matrix $w(\underline{\mathbf{A}})=\left(w\left(\underline{a_{i j}}\right)\right)$ where $w\left(\underline{a_{i j}}\right)=\frac{1}{2}\left(a_{i j s}-\right.$ $\left.a_{i j I}\right)$.

Definition 13. The absolute value of an interval matrix $\underline{\mathbf{A}}$ is the matrix $|\underline{\mathbf{A}}|=\left(\left|\underline{a_{i j}}\right|\right)$.

According to [18], three criteria can be used to determine the best solution to the linear interval programming problem. These criteria are:

1) satisfy the constraint function

2) The width of the interval from the optimum value (the narrowest)

3) Degree of uncertainty is a ratio between the width of the interval and the midpoint of the interval (smallest). 


\section{MODIFICATION INTERIOR-POINT METHOD}

This interval linear programming solution algorithm is based on the interior point algorithm, which is then modified. Modifications are intended to conform to the definitions and theorems that apply to the interior point method, interval operations, and the problem's constraints to be solved. The steps for solving interval linear programming based on interval arithmetic are as follows.

Step 1: Problem

Maximize (objective function)

$\underline{Z}=\sum_{j=1}^{n} \underline{c_{j}} \underline{x_{j}}$

subject to

$\sum_{j=1}^{n} a_{i j} x_{j} \leq \underline{b_{i}}, \quad i=1,2, \ldots, m$,

$\underline{x_{j}} \geq 0, \quad j=1,2, \ldots, n$.

and $x_{j} \in I\left(\mathbb{R}^{+}\right), \underline{c_{j}}, \underline{a_{i j}}, \underline{b_{i}} \in I(\mathbb{R})$.

Step 2: Forming the problem in Step 1 into standard interval linear programming form Maximize (objective function)

$\underline{\mathbf{Z}}=\underline{\mathbf{C}^{\mathrm{T}} \mathbf{X}}$,

subject to

$\underline{\mathbf{A X}}=\underline{\mathbf{b}}$,

$\underline{\mathbf{X}} \geq \underline{\mathbf{0}}$

and

$\underline{\mathbf{C}}=\left[\begin{array}{c}\frac{c_{1}}{c_{2}} \\ \hline \vdots \\ \frac{c_{n}}{0} \\ \underline{0} \\ \underline{0} \\ \vdots \\ \underline{0}\end{array}\right], \underline{\mathbf{X}}=\left[\begin{array}{c}\frac{x_{1}}{x_{2}} \\ \vdots \\ \underline{x_{n}} \\ \underline{x_{n+1}} \\ \underline{x_{n+2}} \\ \frac{\vdots}{x_{n+m}}\end{array}\right], \underline{\mathbf{b}}=\left[\begin{array}{c}\frac{b_{1}}{b_{2}} \\ \frac{b_{2}}{\vdots} \\ \underline{b_{m}}\end{array}\right], \underline{\mathbf{0}}=\left[\begin{array}{c}\frac{0}{0} \\ \underline{0} \\ \underline{0} \\ \underline{0} \\ \underline{0} \\ \vdots \\ 0\end{array}\right]$,

$\underline{\mathbf{A}}=\left[\begin{array}{cccccccc}\frac{a_{11}}{a_{21}} & \frac{a_{12}}{a_{22}} & \cdots & \frac{a_{1 \mathrm{n}}}{a_{2 \mathrm{n}}} & \underline{1} & \underline{0} & \cdots & \underline{0} \\ \hdashline \vdots & \frac{1}{a_{2}} & \ddots & \frac{1}{\vdots} & \underline{\vdots} & \cdots & \ddots & \underline{0} \\ \underline{a_{m 1}} & \underline{a_{m 2}} & \cdots & a_{m n} & \underline{0} & \underline{0} & \cdots & \underline{1}\end{array}\right]$,

where $\underline{\mathrm{C}}, \underline{X}, \underline{0} \in \mathrm{I}\left(\mathbb{R}^{n+m}\right)$, $\underline{\mathrm{b}} \in \mathrm{I}\left(\mathbb{R}^{m}\right)$, and $\underline{\mathrm{A}} \in \mathrm{I}\left(\mathbb{R}^{m \times(n+m)}\right)$.
Step 3: Determine any initial interior-point $\underline{\tilde{\mathbf{X}}^{0}}=$ $\left(x_{1}, \underline{x_{2}}, \ldots, \underline{x_{n+m}}\right)$ that satisfies the constraints in Equation (5) and calculate the value of $\mathbf{Z}_{0}$, with

$\mathbf{Z}_{\mathbf{0}}=\underline{\mathbf{C}^{\mathbf{T}} \tilde{\mathbf{X}}^{\mathbf{0}}}$,

and $\underline{x_{1}}, \underline{x_{2}}, \ldots, \underline{x_{n+m}} \in \mathrm{I}(\mathbb{R}), \underline{x_{1}}, \underline{x_{2}}, \ldots, \underline{x_{n+m}}>0$.

Step 4: Determine the diagonal matrix

$\underline{\mathbf{D}_{i+1}}=\operatorname{diag}\left(\underline{\tilde{\mathbf{x}}^{i}}\right)=\left[\begin{array}{ccccc}x_{1} & \mathbf{0} & \mathbf{0} & & \mathbf{0} \\ \mathbf{0} & \frac{x_{2}}{0} & \mathbf{0} & \cdots & \mathbf{0} \\ \mathbf{0} & \mathbf{0} & \underline{x_{3}} & \ldots & \mathbf{0} \\ \vdots & \vdots & \vdots & \ddots & \vdots \\ \mathbf{0} & \mathbf{0} & \mathbf{0} & \cdots & \underline{x_{n+m}}\end{array}\right]$

Step 5: Determine

$\left.\begin{array}{l}\underline{A_{i+1}}=\underline{\mathbf{A D}_{i+1}} \\ \underline{\mathrm{C}_{i+1}}=\underline{\mathrm{D}_{i+1}} \underline{\mathrm{C}}\end{array}\right\}$

Step 6: Determine

a) Projection matrix

$\underline{\mathbf{P}_{i+1}}=\underline{I}-\underline{\mathbf{A}_{i+1}{ }^{T}\left(\mathbf{A}_{i+1} \mathbf{A}_{i+1}{ }^{T}\right)^{-1} A_{i+1}}$

with I identity matrix.

b) Projected gradient level

$\underline{\mathrm{C}_{P_{i+1}}}=\underline{\mathbf{P}_{i+1} \mathrm{C}_{i+1}}$.

Step 7: Determine

$$
\underline{\mathbf{V}_{i+1}}=\left|\min \left(\underline{\mathbf{C}_{P_{i+1}}}\right)\right|
$$

$\left.\underline{\mathbf{M}_{i+1}}=\left[\begin{array}{c}{[1,1]} \\ {[1,1]} \\ \vdots \\ {[1,1]}\end{array}\right]+\underline{\underline{\mathbf{V}_{i+1}}} \underline{\mathbf{C}_{P_{i+1}}}\right\}$

where $\underline{\mathbf{M}}_{i+1} \in \mathrm{I}\left(\mathbb{R}^{n+m}\right)$ and $\alpha \in(0,1)$.

Step 8: Determine the candidate interior points for the next iteration, i.e.

$\underline{\tilde{\mathrm{X}}^{i+1}}=\underline{\mathrm{D}_{i+1} \mathrm{M}_{i+1}}$

Interior point candidate inspection (non-negative test and constraint boundary test)

a) If the candidate interior point satisfies the constraint limit, proceed to Step 9

b) If the candidate interior point does not meet the constraint limit, then stop. Return to the previous iteration and take the last interior point of the interval that satisfies the constraint limit; proceed to Step 9 
Step 9 : Determine the optimum value

$$
\underline{\mathrm{Z}_{i+1}}=\underline{\mathrm{C}^{\mathrm{T}} \tilde{\mathrm{X}}^{i+1}}
$$

Optimum value check (optimality test)

a) If $\mathbf{Z}_{i+1}>\underline{\mathbf{Z}_{i}}$, then proceed to the next iteration with the same steps as in Step 1-Step 8 in the previous iteration. $\underline{\tilde{\mathbf{X}}^{i+1}}$ is chosen to be the interior point for that iteration.

b) If $\underline{\mathbf{Z}_{i+1}} \leq \underline{\boldsymbol{Z}_{i}}$, then stop, proceed to Step 10 .

Step 10 : The optimum solution is obtained, namely the optimum point and the optimum value in the form of an interval

$\underline{x_{i}}=\left[x_{i I}, x_{i S}\right], i=1,2, \ldots, m$, and $\underline{Z}=\left[z_{I}, z_{S}\right]$.

\section{NUMERICAL EXAMPLE}

In this section, we solve one example of interval linear programming in $[15,18,19,20]$ and compare the results.

Maximize (objective function)

$Z=[26,30] \underline{x_{1}}-[5.5,6] \underline{x_{2}}$

subject to

$$
\left.\begin{array}{c}
{[8,10] \underline{x_{1}}-[12,14] \underline{x_{2}} \leq[3.8,4.2]} \\
[1,1.1]] \underline{x_{1}}+[0.19,0.2] \underline{x_{2}} \leq[6.5,7] \\
\underline{x_{1}}, \underline{x_{2}} \geq 0
\end{array}\right\}
$$

Forming the problem in Equation (16) into standard interval linear programming form

Maximize (objective function)

$Z=[26,30] \underline{x_{1}}-[5.5,6] \underline{x_{2}}+0 \underline{S_{1}}+0 \underline{S_{2}}$

subject to

$$
\left.\begin{array}{c}
{[8,10] \underline{x_{1}}-[12,14] \underline{x_{2}}+\underline{S_{1}}=[3.8,4.2]} \\
{[1,1.1] \underline{x_{1}}+[0.19,0.2] \underline{x_{2}}+\underline{S_{2}}=[6.5,7]} \\
\underline{x_{1}}, \underline{x_{2}}, \underline{S_{1}}, \underline{S_{2}} \geq \underline{0}
\end{array}\right\}
$$

An initial interior interval is taken, which satisfies the constraint in Equation (19) i.e. $\underline{\widetilde{\mathbf{X}}^{0}}=([2,2],[5$, $5],[51,51],[3.5,3.5])$, the value obtained is $\mathbf{Z}_{0}=$ $[22,32.5]$. By using Octave16 software, the optimum solution is $\underline{x_{1}} \cong[3.8109,4.9579], \underline{x_{2}} \cong[2.3034$, 5.7705] and $\underline{Z} \cong[64.462,136.07]$. The results of the optimum point and the optimum value can be seen in Table 1, while Table 2 presents comparison criteria between all solutions.
From Table 1, it can be seen, the solution satisfies nine properties of Definition 9 and is better than $[18,19,20]$ if using the constraint value criterion. If it refers to the interval width criteria, the solution is better than [19]. (2009). Meanwhile, when referring to the requirements for the degree of uncertainty, [15] solution using the modification simplex method is the best. These results can be identified as follows: (1) There is difficulty in taking the initial interior interval [9]. (2) Calculation of the inverse interval still uses the approach [22].

From the calculation example above, solving the interval linear programming problem can use the interiorpoint method based on interval arithmetic calculations to improve taking the initial interior interval and determining the appropriate inverse matrix.

\section{CONCLUSIONS}

The solution of interval linear programming can involve all interval components directly. It is called interval linear programming based on interval arithmetic. The first step in solving interval linear programming problems is to form into standard interval linear programming. Standard interval linear programming is a classical standard linear programming modified by substituting the point elements in intervals. It begins with determining the initial interior interval that satisfies the problem constraints. Then modify the interior point method by changing the points into intervals to solve the problem until the optimum point and optimum value are obtained in the interval. The difficulty of solving interval linear programming that directly involves all interval components is in determining the initial interior interval and the inverse of the interval matrix. Sometimes this difficulty results in the solution obtained is not the best solution compared to the settlement method used by previous researchers. 
Table 1. The optimum point and the optimum value

\begin{tabular}{|c|l|c|c|}
\hline No & \multicolumn{1}{|c|}{ Researchers } & $\underline{\boldsymbol{x}_{\boldsymbol{j}}}$ & $\underline{\boldsymbol{Z}}$ \\
\hline 1 & Huang (1992) [18] & $\underline{x_{1}} \cong[5.21,6.34] ; \underline{x_{2}} \cong[3.32,4.03]$ & $\underline{Z} \cong[111.4,171.8]$ \\
\hline 2 & Zhou et al. (2009) [19] & $\underline{\underline{x_{1}} \cong[4.574,6.336] ; \underline{x_{2}} \cong[3.320,3.495]}$ & $\underline{Z} \cong[97.954,171.82]$ \\
\hline 3 & Suprajitno (2010) [15] & $\underline{x_{1}} \cong[3.7,4.1] ; \underline{x_{2}} \cong[2.7,3.1]$ & $\underline{Z} \cong[77.6,108.15]$ \\
\hline 4 & Fan \& Huang (2012) $[20]$ & $\underline{x_{1}} \cong[5.21,6.23] ; \underline{x_{2}} \cong[3.26,4.03]$ & $\underline{Z} \cong[111.38,169.1]$ \\
\hline 5 & Agustina et al. [now] & $\underline{x_{1}} \cong[3.8109,4.9579] ; \underline{x_{2}} \cong[2.3034,5.7705]$ & $\underline{Z} \cong[64.462,136.07]$ \\
\hline
\end{tabular}

Table 2. Comparison criteria solutions

\begin{tabular}{|c|c|c|c|c|c|}
\hline \multirow[b]{2}{*}{ Solutions } & \multicolumn{5}{|c|}{ Criteria } \\
\hline & Constraint value 1 & Constraint value 2 & $\begin{array}{l}\text { Width of } \\
\text { interval }\end{array}$ & $\begin{array}{c}\text { Midpoint of } \\
\text { interval }\end{array}$ & $\begin{array}{c}\text { Degree of } \\
\text { uncertainty }\end{array}$ \\
\hline Huang [18] & {$[-14.74,23.56] \leq[3.8,4.2]$} & {$[5.8408,7.78] \leq[6.5,7]$} & 30.2 & 141.6 & $21.3276 \%$ \\
\hline Zhou et al. [19] & {$[-12.338,23.52] \leq[3.8,4.2]$} & $\begin{array}{l}{[5.2048,7.6686]} \\
\leq[6.5,7]\end{array}$ & 36.933 & 134.887 & $27.3807 \%$ \\
\hline Suprajitno [15] & {$[-13.8,8.6] \leq[3.8,4.2]$} & {$[4.213,5.13] \leq[6.5,7]$} & 15.275 & 92.875 & $16.4468 \%$ \\
\hline Fan \& Huang [20] & {$[-14.74,23.18] \leq[3.8,4.2]$} & {$[5.8294,7.659] \leq[6.5,7]$} & 28.86 & 140.24 & $20.579 \%$ \\
\hline Agustina et al. [now] & $\begin{array}{l}{[-50.2998,21.9382]} \\
\leq[3.8,4.2]\end{array}$ & $\begin{array}{l}{[6.612091,8,621515]} \\
\leq[6.5,7]\end{array}$ & 35.804 & 100.266 & $35.709 \%$ \\
\hline
\end{tabular}

\section{ACKNOWLEDGMENTS}

This project is supported by a Post-Doctoral Research Grant from the Jember University with Contract No 3479/UN25.3.1/LT/2020

\section{REFERENCES}

[1] H.A. Taha,_Operation Research : An Introduction, Ed. $8^{\text {th }}$, New Jersey,Pearson Prentice Hall, 2007.

[2] F.S. Hillier, G.J. Lieberman, Introduction to Operation Research, Ed. 10 ${ }^{\text {th }}$. McGraw-Hill, Inc., New York, 2010.

[3] N. Karmakar, A New Polynomial Time Algorithm for Linear Programming, Combinatorica, 4(4), 1984, pp 373-395. DOI:https://doi.org/10.1007/BF02579150

[4] D. den Hertog, Interior Point Approach to Linear, Quadratic and Convex Programming, Springer Science+Business Media, BV, 1994. DOI:https://doi.org/10.1007/978-94-011-1134-8

[5] J. Gondzio, Interior Point Methods 25 Years Later, European Journal of Operational Research, 218(3), 2012 , pp. 587-601. DOI:https://doi.org/10.1016/j.ejor.2011.09.017

[6] O. Caprani, K. Madsen, H. Nielsen, Introduction to Interval Analysis, Annual Report Year. DTU Orbit - The Research Information System. IMM-DTU. Technical University of Denmark, 2002.
[7] R.E. Moore, Interval Analysis. Prentice-Hall, Englewood Cliffs, NJ, 1966.

[8] T. Shaocheng, Interval Number and Fuzzy Number Linear Programmings, Fuzzy Sets And Systems, 66(3), 1994, pp. 301-306. DOI:https://doi.org/10.1016/0165-0114(94)90097$\underline{3}$

[9] J.W. Chinnek, K. Ramadan, Linear Programming with Interval Coefficients, Journal of The Operational Research Society, 51(2), 2000, pp. 209220.

DOI:https://doi.org/10.1057/palgrave.jors.2600891

[10] D. Kuchta, A Modification of A Solution Concept of The Linear Programming with Interval Coefficients in The Constraints, Central European Journal of Operations Research, 16(3), 2008, pp. 307-316. DOI:https://doi.org/10.1007/s10100-0080059-X

[11] A. Pradjaningsih, Fatmawati, H. Suprajitno, Interior Point Method for Solving Linear Programming with Interval Coeficients Using Affine Scalling, Far East Journal of Mathematical Sciences, 111(2), 2019, pp. 195-211.

DOI:https://doi.org/10.17654/MS111020195

[12]H. Suprajitno, I. bin Mohd, Interval Linear Programming, in Proc. of the 3th International Conference on Mathematics and Statistics, 2008.

[13] H. Suprajitno, I. bin Mohd, M. Mamat, F. Ismail, Linear Programming With Interval Variables, in Proc. of ICORAFSS, 2009. 
[14] A. Pradjaningsih, Fatmawati, H. Suprajitno, A Solution of Linear Programming with Interval Variables Using The Interior Point Method Based on Interval Boundary Calculations, Far East Journal of Applied mathematics, 109(2), 2021, pp. 103-119. DOI:https://doi.org/10.17654/AM109020103

[15] H. Suprajitno, I. bin Mohd, Linear Programming with Interval Arithmetic, International Journal Contemporary Mathematical Sciences. 5(7), 2010, pp. 323-332.

[16] G. Ramesh, K. Ganesan, Interval Linear Programming with Generalized Interval Arithmetic, International Journal of Scientific \& Engineering Research, 2(11), 2011, pp. 1-8.

[17] G. Ramesh, K. Ganesan, Duality Theory for Interval Linear Programming, IOSR Journal of Mathematics 4(4), 2012, pp. 39-47. DOI:https://doi.org/10.9790/5728-0443947

[18] G.H. Huang, B.W. Baetz, G.G. Patry, A Grey Linear Programming Approach for Municipal Solid Waste
Management Planning Under Uncertainty, Civil Engineering Systems. 9(4): 1992, pp. 319-335. DOI:https://doi.org/10.1080/02630259208970657

[19] F. Zhou, G.H. Huang, G. Chen, H. Guo, EnhancedInterval Linear Programming, European Journal of Operational Research, 199(2), 2009, pp. 323-333. DOI:https://doi.org/10.1016/j.ejor.2008.12.019

[20] Y.R. Fan, G.H. Huang, A Robust Two-Step Method for Solving Interval Linear Programming Problems within An Environmental Management Context, Journal of Environmental Informatics, 19(1): 2012, pp. 1-9. DOI:https://doi.org/10.3808/jei.201200203

[21] G. Alefeld, J. Herzberger, Introduction to Interval Computation, Academic Press, Inc., New York, 1983.

[22] T. Nirmala, D. Datta, H.S. Kushwaha, K. Ganesan, Inverse Interval Matrix: A New Approach. Applied Mathematical Sciences, 5(13), 2011, pp. 607 - 624. 